\title{
BMJ Open Menstrual experience of adolescents in the USA: protocol for a scoping review
}

\author{
Ronke Olowojesiku (1) , Deborah J Shim, Bryanna Moppins, Daye Park, \\ Jasmine O Patterson, Samantha A Schoenl, Julie K Gaines, Edwin V Sperr, \\ Amy Baldwin
}

To cite: Olowojesiku R, Shim DJ, Moppins B, et al. Menstrual experience of adolescents in the USA: protocol for a scoping review. BMJ Open 2021;11:e040511. doi:10.1136/ bmjopen-2020-040511

- Prepublication history for this paper is available online. To view these files, please visit the journal online (http://dx.doi org/10.1136/bmjopen-2020040511).

Received 03 June 2020 Revised 11 January 2021 Accepted 23 January 2021

Check for updates

(c) Author(s) (or their employer(s)) 2021. Re-use permitted under CC BY-NC. No commercial re-use. See rights and permissions. Published by BMJ.

AU/UGA Medical Partnership, Athens, Georgia, USA

Correspondence to Dr Ronke Olowojesiku; rolowoj@gmail.com

\section{ABSTRACT}

Introduction In recent years, there has been a growing desire to address issues related to menstruation,

particularly for adolescent girls. In low-income and middleincome countries, prior literature review of the adolescent menstrual experience suggests the need for further research into the impact and efficacy of interventions with this population. There is evidence to suggest the need for initiatives and research in higher-income countries like the USA. To date, the body of research on adolescent menstrual experience in the USA remains uncharacterised. Therefore, we propose a scoping review of the literature on this subject to better inform on areas for future primary study.

Methods and analyses Using the framework proposed by Arksey and 0'Malley and expounded on by Levac et al and the Joanna Briggs Institute, we will search electronic databases (MEDLINE, CINAHL, PsycINFO, Web of Science, ProQuest Public Health Database, Social Science Citation Index, Social Services Abstracts and SocINDEX) and grey literature for relevant studies in consultation with experienced librarians. The abstracts and full-text from each reference will be screened by two independent reviewers for inclusion. Bibliographic data, study characteristics and themes will be extracted from studies selected for inclusion using a rubric created by the research team. Findings will be summarised and a list of subject areas for future primary research will be generated in consultation with stakeholders. The review will be conducted using the Preferred Reporting Items from Systematic Reviews and Meta-Analyses extension for Scoping Reviews guidelines.

Ethics and dissemination Formal ethics training for this study is not required, as the research team will review publicly available studies. Stakeholders working in adolescent and menstrual health were consulted in designing this review. We will share key findings with stakeholders and in scholarly journals at the conclusion of the review.

\section{INTRODUCTION}

Women of reproductive potential aged $15-50$ comprise about one-quarter of the US population. ${ }^{12}$ This period of reproductive potential is critical for both the health of the woman and any children she may bear. Management of reproductive health issues remains a challenge for women of reproductive age in the

\section{Strengths and limitations of this study}

This review will provide insight into a critical knowledge gap, as there is a lack of synthesis of the research available on the menstrual experiences of adolescents in the USA. Through the scoping review process, we will describe the current body of evidence available on this subject to inform future primary study and contribute to a broad understanding of the topic.

- We propose an extensive, comprehensive search strategy of both published literature from multiple databases and grey literature to maximise the amount of research captured.

- To ensure relevant and accurate adolescent-centred data, we incorporate information from various stakeholders that are knowledgeable and/or work closely with the target demographic to assist in the framing of the research question and the drawing of conclusions from the retrieved data.

- This review is limited to English-language studies published in the last 20 years; although this will not result in a complete review of the literature, we anticipate that the bulk of studies on this subject will have been published in this time frame and in English given our country of study. This review is limited to one country of study, and therefore, may not be generalisable to other similar settings, but we anticipate it will provide rich data for future study. Additionally, while we attempt to conduct a broad search of published and grey literature, it is possible that our review may not fully capture the body of evidence on this subject.

USA, with disparities noted across racial/ ethnic, socioeconomic and regional lines. ${ }^{34}$ In considering some markers of reproductive health, the USA has higher rates of unplanned pregnancy and maternal mortality compared with other industrialised nations. ${ }^{5}{ }^{6}$ While reproductive health research covers a wide array of topics, less work has been done to understand the various factors related to another key component of health: menstruation and menstrual health. ${ }^{7}$

Menstruation is a monthly marker of a woman's reproductive potential. Despite 
its ubiquity, issues related to menstruation remain unaddressed or underaddressed, including the area of menstrual education. ${ }^{78}$ In a survey of 125 women in the USA, $52.8 \%$ of participants expressed knowledge of the ovulatory cycle and $50.4 \%$ knew the average length of a menstrual period. ${ }^{9}$ In a larger survey of 1000 women in the USA of various ethnic, socioeconomic and educational backgrounds, about $40 \%$ of respondents demonstrated an incomplete understanding of the ovulatory cycle, and knowledge gaps in contraception and infertility risk factors were identified. ${ }^{10}$ Additionally, the authors noted significant differences in knowledge gaps by age, with more gaps being present among younger women aged $18-24 .{ }^{10}$ These findings have far-reaching implications on well-being, particularly if evidence suggests greater knowledge gaps in younger women, including adolescent girls.

Adolescence is an important time in a young person's life, especially for young women. These prepubertal and peripubertal years are a time when women build an understanding of their sexual selves and can make decisions that may impact their long-term reproductive and sexual health. ${ }^{11}$ Limited understanding of menarche and menstruation can contribute to misunderstandings surrounding the process of fertilisation and pregnancy, and poor menstrual hygiene leaves one at risk of urinary and reproductive tract infections that can have lasting implications. ${ }^{11}$ Examples of interventions and initiatives to promote menstrual health and education are typically emphasised in low-income and middle-income countries (LMICs), such as programmes for reusable pads and the Water, Sanitation and Hygiene (WASH) curriculum for menstrual hygiene and education. ${ }^{12}{ }^{13}$ Studies conducted on the menstrual experiences of adolescents in LMICs, particularly a recent scoping review by Coast $e t a l$, highlight the limited understanding of puberty and menstruation among adolescents and the need for more robust evidence to evaluate the consequences of menstrual stigma on a young girl's overall well-being and the efficacy of ongoing interventions in these settings. ${ }^{1415}$

Further evidence suggests that menstruation issues in adolescence are not solely confined to LMICs. Plan International UK reports in a survey of 1000 girls and young women aged 14-21 living in England and Northern Ireland that one in seven girls did not understand what was occurring in their bodies at the start of menses. ${ }^{16}$ Additionally, one in four felt unprepared for menarche. ${ }^{16}$ Girls overall reported feelings of stigma, with almost half stating they felt embarrassed by their period. ${ }^{16}$ Along with the emotional distress, the survey found evidence of financial issues, with 1 in 10 girls being unable to afford menstrual products, and school absenteeism, with $49 \%$ of girls missing at least 1 day of school due to their period. ${ }^{1617}$ Limited community studies done by individual groups in the USA as well as an earlier review of teen puberty experiences reflect similar findings, suggesting that many populations of girls in the States have comparable menstrual experiences to those of their peers in LMICs with regard to their access to menstrual products and understanding of menstruation overall. ${ }^{1819}$

Work remains to be done with regard to research on the adolescent menstrual experience in the USA, as highlighted by advocates in the field; indeed there has been a recent call for increased efforts to generate evidence on this important health topic. ${ }^{7820}$ Experts have also called for the need to normalise conversation around menstruation as to better address the issues that remain. ${ }^{20}$ In sum, the unwillingness to discuss menstruation negatively impacts the lives of young girls worldwide, especially in the USA, where the overall wealth of the nation can cause vulnerable populations to be further overlooked and marginalised.

\section{STUDY RATIONALE}

There are growing efforts to address issues related to menstruation by various advocacy groups and menstrual product companies, as well as calls to action on menstrual needs from agencies such as WHO. ${ }^{21-23}$ Studies globally on issues such as access to menstrual and feminine hygiene products have shown that unmet menstrual needs negatively impact young girls, from missed days in school to shame from family and community members. ${ }^{14} 1517$ Many of these studies have been conducted in LMICs, thus the particular menstrual health needs of adolescents in the USA has not been fully explored. We chose to focus on the USA specifically as opposed to other high-income countries given its healthcare landscape and the disparities within the society. The multisystem, non-universal healthcare model present in the States poses unique challenges that leave more individuals without regular access to health services than in other similar countries. ${ }^{24}$ Additionally, when compared with other Organisation for Economic Co-Operation and Development (OECD) nations, the US boasts the second highest poverty rate and is among the nations with high rates of child poverty. ${ }^{25}{ }^{26}$ Given the significant proportion of individuals in the USA that live in poverty, particularly in the study age range, and the limitations of the healthcare system to provide regular services to all individuals, it is possible that adolescents in the States may have more issues with access to menstrual health products and services compared with their peers in other economically similar countries. These potential issues may ultimately impact their overall menstrual experience and thus warrants specific attention. Understanding the research conducted to date on the menstrual experiences of adolescents in the USA is essential to better advocate for changes to meet unmet needs. By highlighting the work that has been completed in this area, we hope to demonstrate how limited the body of evidence is, thereby having a basis to provide directions for future primary research.

\section{OBJECTIVES}

Given the anticipated heterogeneous nature of the research subject at hand, we aim to (1) systematically review the literature on adolescent menstrual experiences using the scoping review process, (2) characterise this body of literature as it 
relates to adolescent girls in the USA by study characteristics, population and subject area, and (3) identify gaps in the literature for future primary study. Research studies selected for analysis will be assessed for relevance through a selective screening process involving consensus between independent reviewers. The scoping review will streamline available research on adolescent menstrual experience within our selected inclusion criteria. This information will allow healthcare professionals and relevant community organisations to assess the current status of the delivery and understanding of adolescent menstrual health as well as expand resources and research in areas that require improvement.

\section{METHODS AND ANALYSES}

We use methods for the scoping review as described by Arksey and O'Malley and further expanded on by Levac et al, and the Joanna Briggs Institution (JBI) ${ }^{27-29}$ The scoping review approach was selected given the heterogeneous nature of the research done in this area. Based on the framework proposed by Arksey and O'Malley, the review will be conducted in the following six stages: (1) identification of the research question, (2) identification of relevant studies, (3) study selection, (4) data charting, (5) collation, summarisation and report of results, and (6) consulting with relevant stakeholders. ${ }^{24}$ The review will follow the Preferred Reporting Items for Systematic Reviews and Meta-Analyses Extension for Scoping Reviews. ${ }^{30}$

\section{Identifying the research question}

After consultation with stakeholders working with adolescents and menstrual health, our research team developed the following comprehensive research question: 'What is the current body of research on the experiences of menstruation among adolescents in the USA in relation to their race/ ethnicity, urbanicity, regionality, socioeconomic status and education level?' We have defined menstrual experience to include knowledge, thoughts, feelings, beliefs, narratives and practices related to menarche and menstruation, issues related to access to menstrual care products and impact of menstruation on daily living.

\section{Identifying relevant studies \\ Inclusion criteria}

All available original primary studies, systematic reviews, meta-analyses, conference abstracts and dissertations published between 2000 and 2020 and conducted on human subjects will be considered for inclusion based on the criteria in table 1 , described in the population, concept and context framework proposed by JBI. ${ }^{29}$

\section{Exclusion criteria}

Non-human and non-English studies from before the year 2000 will be excluded.

Regarding the subject area of interest, the following studies will be excluded:

- Studies addressing puberty in general and not specifically menstruation (eg, development of secondary sexual characteristics).

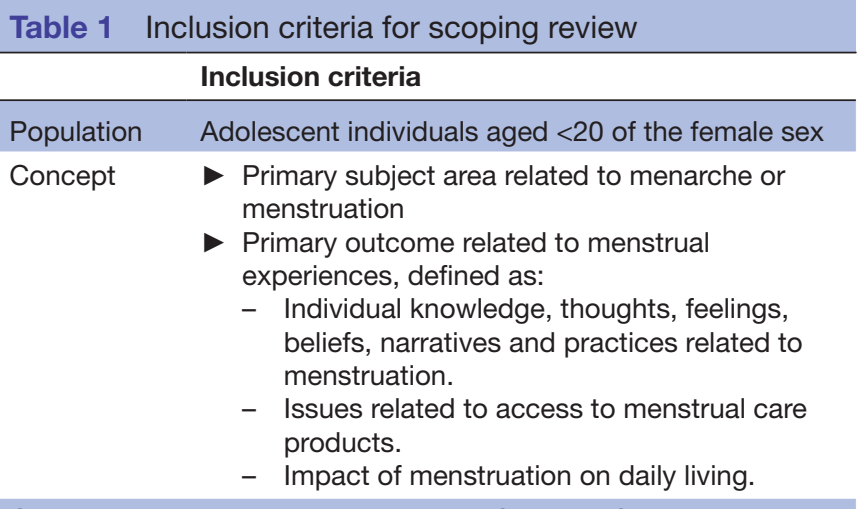

Context Based in present-day USA and US territories

- Studies addressing topics that affect menstruation but do not address menstruation as a primary area of study (eg, female athlete triad, disordered eating and amenorrhoea, developmental or behavioural comorbidities and their impact on menstrual health and hygiene).

- Studies in which the adolescent perspective regarding the menstrual topic area is not explicitly studied (eg, parent/guardian opinion, clinical opinion/ guideline).

\section{Search strategy}

Relevant studies for the scoping review will be identified and retrieved by searching the following electronic bibliographic databases: MEDLINE, Cumulative Index to Nursing and Allied Health Literature (CINAHL), PsycINFO, Web of Science, ProQuest Public Health Database, Social Science Citation Index, Social Services Abstracts and SocINDEX. An example of the proposed search strategy for PubMed is outlined in online supplemental appendix A. The team will also search the reference lists of selected studies to identify other materials to be included.

To ensure all pertinent information is captured for the review, the team will also search for grey literature by identifying relevant governmental and non-governmental organisations that work in this field. Through searching their sites and conference programmes, we will identify additional organisational and government reports, conference abstracts and studies related to our search topic. Additional reports will be added based on expert review and recommendation.

The two academic librarians on the team worked closely with other team members and subject experts to identify search keywords and controlled vocabulary headings to incorporate into the searches for each database. In keeping with the inclusion criteria, searches will be limited to materials published during the years 2000-2020.

\section{Study selection}

References retrieved from database search will be added to an Endnote library for screening. To identify articles to include in the review, we will implement a two-stage 
screening process at the level of the title and abstract and full text using the criteria outlined above. References will be managed during the screening process using Endnote and the Rayyan web-based application. ${ }^{31}$ A random sample of references to be screened will be selected as a trial of the selection criteria to ensure comparable application of criteria among reviewers prior to the start of the screening process. At the completion of the screening process, all reference lists of selected studies will be hand searched to identify additional relevant studies as resources allow.

\section{Title and abstract screen}

Two reviewers will independently screen the title, abstract and subject headings of references identified in the search using Rayyan. References will be assigned the following final decisions: yes-include, no-exclude or unclear. Studies marked as yes or unclear will be included for full-text review. Any disagreements between the reviewer pairs will be resolved by consensus. If consensus cannot be reached, a third independent reviewer will serve as the tiebreaker. A random sample of $5 \%$ of the references screened will be reviewed by a third reviewer to ensure quality of the title and abstract screen.

\section{Full text review}

References marked as yes or unclear will be considered for full-text review. Two independent reviewers will screen references for inclusion using the same criteria from the title and abstract screen. References will be marked as either yes-include or no-exclude. Any disagreements between reviewers will be resolved by consensus and if required, by decision of a third reviewer. Reasons for exclusion will be documented. A random sample of $5 \%$ of references screened at the full-text stage will be reviewed by a third reviewer to ensure the quality of the full-text review. The number of articles that could not be retrieved for full-text review will be documented.

\section{Data charting}

Data charting describes the process of sorting information into key topic areas and themes. ${ }^{27} \mathrm{~A}$ standardised form will be created to chart data. The form will be piloted with a random sample of studies selected for inclusion and refined in an iterative manner. Once the form is finalised, data will be extracted in duplicate by two independent reviewers. Information to be extracted include the following: reference information, study characteristics, study participants, menstrual topic area. Details on the specific variables to be extracted can be found in box 1 .

Disagreements in data charting will be resolved by reviewer consensus or tiebreaker by a third reviewer. To ensure inter-reviewer reliability, a Cohen kappa statistic will be calculated for each reviewer pair, with a goal kappa of greater than or equal to 0.8 . This will be done by creating a two-by-two table of individual reviewer charting (eg, if charted data matched across metrics between individuals in one reviewer pair).

\section{Box 1 Data to be extracted from studies in review}

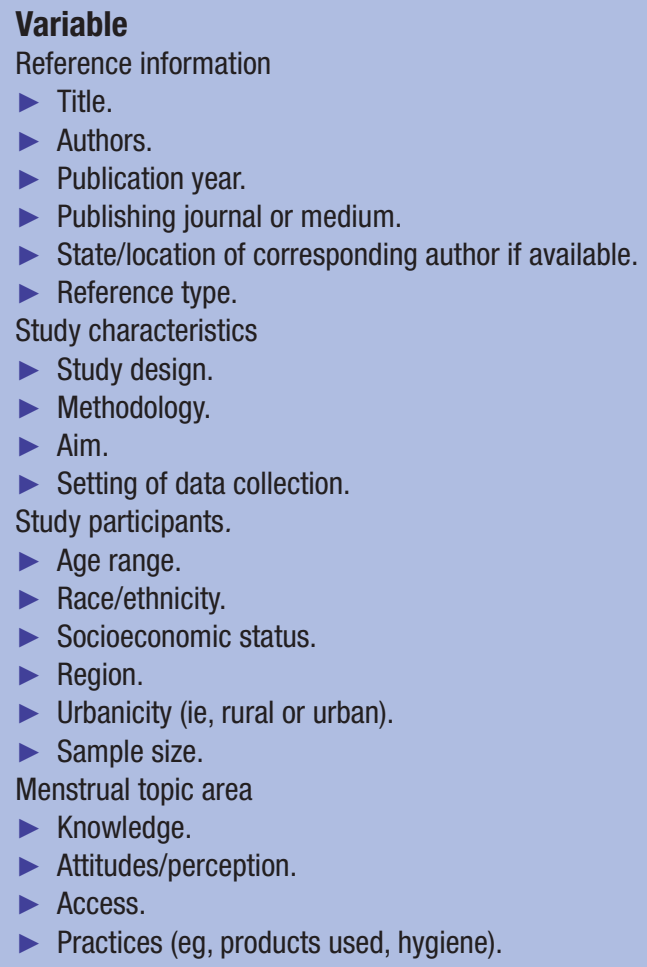

As an assessment of study quality is not required for the scoping review process, one will not be conducted. We are interested in documenting all studies on adolescent menstrual knowledge, attitudes, perceptions and experiences regardless of study quality.

\section{Collation, summarisation and report of results}

Once data is charted, it will be analysed and compiled for presentation. The distribution of study characteristics, study participant characteristics and menstrual topic area will be reported. The aims of the studies will be thematically coded and presented. Key findings from each study may be reported depending on the literature included for review. A summary of all data findings will be given, highlighting the overarching concepts covered by the papers and drawing conclusions on gaps in the literature that remain to be addressed.

\section{Consultation with stakeholders}

Guidelines on the scoping review process suggest consultation with relevant stakeholders to provide direction and insight not directly evident from relevant literature identified. ${ }^{27-29}$ For this protocol, we have incorporated input from members of community organisations providing menstrual hygiene kits to women and girls in need as well as a professor in public health whose research focuses on adolescent health. These individuals were sought out given their active work on menstrual health in our local community. We will consider the input of these stakeholders and others identified outside our local area, including adolescents and school-based officials, throughout the duration of this review. This input will be sought particularly at the conclusion of the full-text review 
to identify any additional studies to be considered, as well as at the completion of data charting for aid in proposing future research directions.

\section{Patient and public involvement}

No patients were involved in the study design. Our proposal centres on a review of published literature and will not involve the recruitment or assessment of individual patients by our team.

\section{ETHICS AND DISSEMINATION}

As this is a systematic review of publicly available literature, ethics training is not warranted. As described, prior to publication of review findings, we will share results with key stakeholders working with adolescents regarding menstrual health to gather their input on the presentation of our results and the conclusions drawn from them. We intend to share our findings at medical education conferences, community events and through publication in a peer-reviewed journal. This review will bring more awareness to this subject, one that is often ignored, and it will spark future menstrual health research in the USA.

Contributors R0 conceived of the idea of the scoping review. RO, DJS, BM and AB developed the research question. RO, JKG, EVS and AB developed the methods; JKG and EVS developed the search strategy. DJS, BM, DP, JOP and SAS contributed to the methods. RO, DJS, BM, DP, JOP, SAS, JKG, EVS and AB contributed significantly to the drafting and editing of the final manuscript. $A B$ supervised the preparation of the manuscript for submission. All authors have approved the final protocol.

Funding R0 is funded by the United Health Foundation/National Medical Fellowships (UHF/NMF) Diverse Medical Scholars Program (Award Number: Not applicable). UHF/NMF was not involved in the study design or preparation of this protocol.

Competing interests None declared.

Patient consent for publication Not required.

Provenance and peer review Not commissioned; externally peer reviewed.

Supplemental material This content has been supplied by the author(s). It has not been vetted by BMJ Publishing Group Limited (BMJ) and may not have been peer-reviewed. Any opinions or recommendations discussed are solely those of the author(s) and are not endorsed by BMJ. BMJ disclaims all liability and responsibility arising from any reliance placed on the content. Where the content includes any translated material, BMJ does not warrant the accuracy and reliability of the translations (including but not limited to local regulations, clinical guidelines, terminology, drug names and drug dosages), and is not responsible for any error and/or omissions arising from translation and adaptation or otherwise.

Open access This is an open access article distributed in accordance with the Creative Commons Attribution Non Commercial (CC BY-NC 4.0) license, which permits others to distribute, remix, adapt, build upon this work non-commercially, and license their derivative works on different terms, provided the original work is properly cited, appropriate credit is given, any changes made indicated, and the use is non-commercial. See: http://creativecommons.org/licenses/by-nc/4.0/.

ORCID iD

Ronke Olowojesiku http://orcid.org/0000-0002-0514-3671

\section{REFERENCES}

1 US Census Bureau: Population Division. Annual estimates of the resident population for selected age groups by sex for the United
States, states, counties, and Puerto Rico Commonwealth and municipios: April 12010 to July 1 2012; 2013.

2 Monte LM, Ellis RR. Fertility of women in the United States: 2012 population characteristics United States Census Bureau; 2014.

3 ACOG Committee opinion no. 586: health disparities in rural women. Obstet Gynecol 2014;123:384-8.

4 Haider S, Stoffel C, Donenberg G, et al. Reproductive health disparities: a focus on family planning and prevention among minority women and adolescents. Glob Adv Health Med 2013;2:94-9.

5 Finer LB, Zolna MR. Declines in unintended pregnancy in the United States, 2008-2011. N Engl J Med 2016;374:843-52.

6 Lu MC. Reducing maternal mortality in the United States. JAMA 2018;320:1237-8.

7 Phillips-Howard PA, Hennegan J, Weiss HA, et al. Inclusion of menstrual health in sexual and reproductive health and rights. Lancet Child Adolesc Health 2018;2:e18.

8 Sommer M, Schmitt M, Gruer C, et al. Neglect of menarche and menstruation in the USA. Lancet 2019;393:2302.

9 Ayoola AB, Zandee GL, Adams YJ. Women's knowledge of ovulation, the menstrual cycle, and its associated reproductive changes. Birth 2016;43:255-62.

10 Lundsberg LS, Pal L, Gariepy AM, et al. Knowledge, attitudes, and practices regarding conception and fertility: a population-based survey among reproductive-age United States women. Fertil Steril 2014;101:767-74.

11 Sumpter C, Torondel B. A systematic review of the health and social effects of menstrual hygiene management. PLoS One 2013;8:e62004

12 AFRIpads. Available: https://www.afripads.com/

13 WASH United. Available: https://wash-united.org/

14 Chandra-Mouli V, Patel SV. Mapping the knowledge and understanding of menarche, menstrual hygiene and menstrual health among adolescent girls in low- and middle-income countries. Reprod Health 2017;14:30.

15 Coast E, Lattof SR, Strong J. Puberty and menstruation knowledge among young adolescents in low- and middle-income countries: a scoping review. Int J Public Health 2019;64:293-304.

16 Tingle CV, Shailini. Break the Barriers: Girls' Experiences of Menstruation in the UK Plan International UK; 2018.

17 Plan International UK. Break the barriers: our menstrual manifesto. Available: https://plan-uk.org/girls-rights-in-the-uk/break-thebarriers-our-menstrual-manifesto

18 Herbert AC, Ramirez AM, Lee G, et al. Puberty experiences of lowincome girls in the United States: a systematic review of qualitative literature from 2000 to 2014. J Adolesc Health 2017;60:363-79.

19 White LR. The Function of Ethnicity, Income Level, and Menstrual Taboos in Postmenarcheal Adolescents' Understanding of Menarche and Menstruation. Sex Roles 2013;68:65-76.

20 Normalising menstruation, empowering girls. Lancet Child Adolesc Health 2018;2:379.

21 Always Wants to Help \#EndPeriodPoverty to Keep Girls in School \& Confident. Available: https://always.com/en-us/about-us/end-periodpoverty

22 Period: the menstrual movement, 2019. Available: https://www. period.org/

23 World Health Organization. Tackling the taboo of menstrual hygiene in the European region, 2018. Available: http://www.euro.who.int/en/ health-topics/environment-and-health/water-and-sanitation/news/ news/2018/11/tackling-the-taboo-of-menstrual-hygiene-in-theeuropean-region

24 OECD. Health at a glance; OECD indicators. Paris OECD Publishing; 2017. https://doi.org/10.1787/health_glance-2017-en

25 OECD. Poverty rate (indicator); 2021.

26 OECD. OECD family database: child mortality, 2019. Available: http:// www.oecd.org/els/soc/CO_2_2_Child_Poverty.pdf

27 Arksey H, O'Malley L. Scoping studies: towards a methodological framework. Int J Soc Res Methodol 2005;8:19-32.

28 Levac D, Colquhoun H, O'Brien KK. Scoping studies: advancing the methodology. Implement Sci 2010;5:69.

29 Peters MDJ, Godfrey CM, Khalil H, et al. Guidance for conducting systematic scoping reviews. Int J Evid Based Healthc 2015;13:141-6.

30 Tricco AC, Lillie E, Zarin W, et al. PRISMA extension for scoping reviews (PRISMA-ScR): checklist and explanation. Ann Intern Med 2018;169:467-73.

31 Ouzzani M, Hammady H, Fedorowicz Z, et al. Rayyan-a web and mobile app for systematic reviews. Syst Rev 2016;5:210. 